
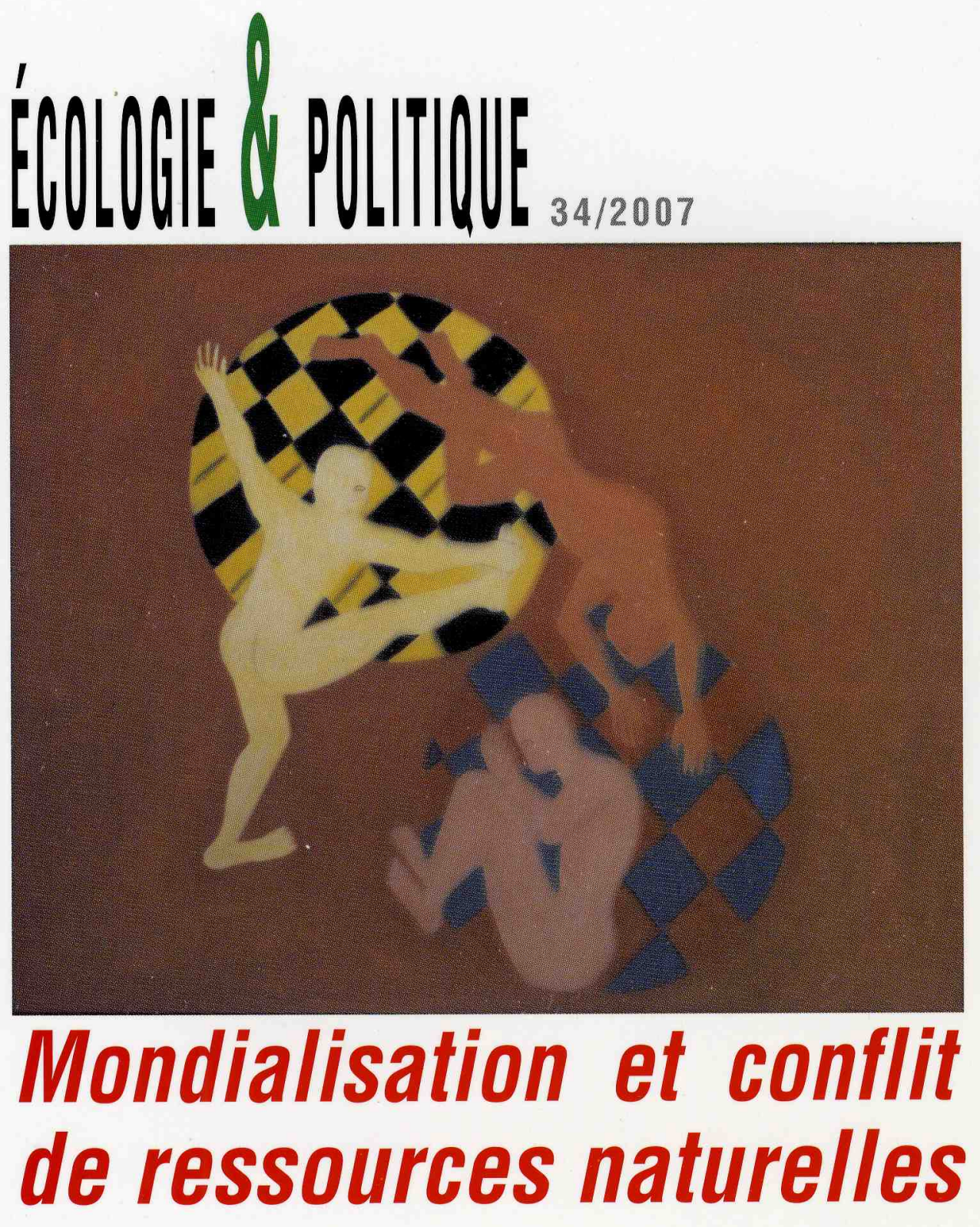

SVLLEPSE 


\section{Mondialisation et conflits autour des ressources halieutiques}

\author{
SylVAIN LE ROUX ET JULIEN NOËL
}

" Guerre de l'anchois ") entre pêcheurs français et espagnols dans le golfe de Gascogne (1992), " guerre du turbot » entre le Canada et l'Espagne près des bancs de Terre-Neuve (1995) ; rivalités entre pêche artisanale et salmoniculture au Chili depuis les années 1990, entre pêches professionnelle et récréative en France en 2005 ; conflits entre pêcheurs artisans et industriels au Sénégal, en Malaisie, au Brésil ; embargos sur le thon entre les ÉtatsUnis et le Mexique (1991), sur la crevette entre la France et Madagascar (1997) ; tensions autour des accords de pêche entre les pays de l'Union européenne (UE) et les pays Afrique-Caraïbes-Pacifique (ACP), etc.

Les ressources halieutiques provoquent des situations conflictuelles à différents niveaux de l'activité, tant dans son exploitation que dans sa gestion. Si ces tensions ne sont pas nouvelles, il s'avère que dans un contexte spatial de plus en plus globalisé et marqué par une raréfaction accrue de la ressource halieutique, ces rivalités tendent à s'exacerber selon des degrés de violence plus ou moins prononcés. De fait, ces ressources communes constituent une source de richesses potentielles qui suscitent de multiples convoitises tout en concentrant des enjeux considérables à divers plans et à diverses échelles ${ }^{[1]}$ : sociaux, avec près de 38 millions d'emplois (directs et induits) à travers le monde ; économiques, avec près de 80 milliards de dollars d'exportations en valeur par an ; alimentaires, avec près de $15 \%$ de la consommation mondiale de protéines animales ; culturels, avec une multitude d'identités locales fortes (traditions, savoirfaire, etc.).

Nous analyserons d'abord les conséquences conflictuelles engendrées par le processus de mondialisation sur la filière halieutique à l'échelle internationale. Puis, à partir de recherches effectuées sur le terrain sénégalais, nous montrerons comment ces recompositions
[1] J.-P. Corlay, « Du poisson pour se nourrir, du poisson pour vivre : les enjeux de la pêche et de l'aquaculture à l'aube du $3 e$ millénaire ", actes du FIG de Saint-Dié, Nourrir les hommes, nourrir le monde. Les géographes se mettent à table, 2004, http://fig-st-die. education.fr/actes/ actes2004/index.htm.

Sylvain Le Roux et Julien Noël sont respectivement docteur et doctorant en géographie à l'université de Nantes au sein du laboratoire de recherche Géolittomer. 
spatiales contribuent à la (re)production de multiples conflits. Enfin, nous verrons quelles sont les diverses propositions pour tenter de réguler durablement cette activité.

\section{La mondialisation, ou les recompositions spatiales conflictuelles de la filière pêche}

Après avoir sommairement définit dans une perspective géographique les notions de mondialisation et de filière halieutique, nous analysons par la suite les principales répercussions spatiales de ce processus sur l'ensemble de ce secteur d'activité.

\section{Le géosystème halieutique}

Pour bien appréhender la structure et l'organisation de la filière halieutique, ainsi que son inscription dans les processus de mondialisation nous nous appuyons sur la méthodologie du géosystème halieutique. Schématiquement, le géosystème représente un système naturel ou artificialisé, constitué de composantes physico-chimiques et bioécologiques, dans lequel les sociétés humaines interviennent (exploitation, distribution, aménagement, etc.) de par leurs pratiques techniques, sociales, culturelles ou économiques. Appliqué au domaine de la pêche par Corlay, ce géosystème résulte « de la rencontre entre un potentiel de ressources biologiques marines », l'écosystème [...] et « d'une stratégie d'exploitation de ce potentiel », le sociosystème. Celui-ci produit « une structure spatiale singulière, l'espace halieutique ${ }^{[2]} »$, qui se décompose en trois volets :

- un espace de production, à l'intérieur duquel les ressources sont prélevées ;

- un espace de distribution, où s'opèrent la commercialisation et la consommation des produits ;

- un espace polarisant, point de jonction structurant entre les deux autres espaces (traitement des produits, administration,...).

\section{Pêches, mondialisation et conflits}

La période des révolutions industrielles marque l'essor de la pro-

[2] J.-P. Corlay, "L'espace halieutique existe, je l'ai rencontré... Essai théorique et méthodologique sur la géographie des pêches », Cahiers Nantais, n40, 1993, p. 59.

[3] Ibid. duction halieutique commerciale et conduit au productivisme et à l'industrialisation du secteur : gigantisme naval, chalutage, congélation,... Le secteur décolle tellement (production multipliée par 5 entre les années 1950 et aujourd'hui) que les premiers signes de surexploitation apparaissent dès les années 1970. Ces « révolutions sectorielles ${ }^{[3]}$ » engendrent des recompositions socio-spatiales contradictoires à l'échelle mondiale et concourent à une certaine « déterritorialisation » de 
ces activités. Le secteur est désormais dominé par quelques puissantes multinationales, aussi bien dans la production (Pescanova en Espagne) que dans la distribution (Findus du groupe suisse Nestlé). Celles-ci organisent la filière à partir de grandes plates-formes, les « super-hubs poissonniers », ce qui engendre des répercussions à tous les niveaux de l'activité.

Ces mutations traduisent tout d'abord une atomisation des grandes zones de production, ainsi que des producteurs. Jusqu' aux années 1980, les eaux froides et tempérées de l'Atlantique Nord concentraient plus de la moitié des captures du globe. Mais suite à une nationalisation juridique des mers $^{[4]}$ et une appropriation territorialisée des zones de pêches ${ }^{[5]}$, l'activité se transfère progressivement vers de nouveaux pays halieutiques riverains des océans Pacifique et Indien, principalement des nations asiatiques (Chine, Inde, Indonésie,...) ou latino-américaines (Pérou, Chili), même si certains pays du Nord (Japon, Russie, ÉtatsUnis, Europe) continuent d'y participer activement. Pourtant, s'il semble y avoir eu un rattrapage des pays du Sud en termes de production sur les pays du Nord au cours des dernières décennies, cela ne s'est pas traduit par une amélioration significative des niveaux de consommation.

Si les échanges de produits de la mer (35\% de la production), multipliés par vingt en 25 ans, représentent un poids conséquent du commerce mondial (60 milliards de dollars par an.), ceux-ci demeurent profondément déséquilibrés. Ils sont révélateurs sur le plan spatial d'une concentration des zones de distribution et de consommation et demeurent le reflet d'une inégalité économique que la mondialisation traduit en inégalité nutritionnelle, à travers un véritable transfert de protéines de la rente aquatique au profit des pays du Nord ${ }^{[6]}$. Pour satisfaire une demande de plus en plus insatiable, ces derniers concentrent près de $80 \%$ des importations et s'approvisionnent sur des espèces ciblées à forte valeur commerciale (crevette, thon, saumon...), alors que le poisson joue un rôle déterminant dans l'alimentation de millions de personnes des pays du Sud (près de $40 \%$ de leur ration protéinique journalière $)^{[7]}$.

Dans un contexte spatial globalisé en permanente recomposition, l'activité halieutique génère de violentes rivalités, elles-mêmes exacerbées par une situation de raréfaction de la ressource et de dégradation du milieu marin. Les conflits territoriaux sont les plus violents ; lorsqu'en mars 1995 un destroyer canadien arraisonne un chalutier espagnol dans les eaux internationales, à 260 milles des côtes de TerreNeuve pour lui interdire de pêcher le turbot, cet incident naval provoque une vive tension entre le Canada et l'UE. Mais des conflits existent également entre types de métier ; en Inde, par exemple, en
[4] Notamment avec la convention des Nations unies sur le droit de la mer, ratifiée en 1982 à Montego Bay.

[5] J. Chaussade et J. Guillaume, Pêche et aquaculture. Pour une exploitation durable des ressources vivantes de la mer et du littoral, Presses universitaires de Rennes, Rennes, 2006.

[6] J. Chaussade, Les ressources de la mer, Flammarion, Évreux, 1997.

[7] A. Le Sann, Du Nord au Sud. Pêcher pour vivre, Crisla, Lorient, 1995. 
1997, les pêcheurs traditionnels travaillant aux moyens d'engins de pêche « passifs » (casiers, palangres) s'opposent violement aux industriels crevettiers étrangers qui utilisent des engins plus « actifs » (chaluts, sennes). Enfin, sur le plan commercial, l'un des conflits le plus révélateur reste celui qui a opposé les États-Unis et le Mexique en 1991, où les États-Unis décrétèrent un embargo sur les importations de thon mexicain prétextant un respect insuffisant de normes environnementales de protection des dauphins de la part des pêcheurs ${ }^{[8]}$.

Bien d'autres exemples pourraient illustrer le caractère conflictuel de l'activité halieutique, notamment celui de la pêche piroguière sénégalaise sur laquelle nous allons insister désormais.

\section{Conflits multiples autour de l'exploitation des ressources halieutiques au Sénégal}

Le Sénégal constitue la troisième puissance halieutique du continent africain. Cette position est liée au dynamisme de la pêche piroguière, qui participe environ à $85 \%$ des 400000 tonnes de produits halieutiques débarqués chaque année au Sénégal ${ }^{[9]}$. Appelée communément "pêche artisanale », cette activité constitue un des principaux piliers de l'économie nationale : première source de devises du pays (près de $38 \%$ des recettes d'exportation en 2001), première activité créatrice d'emplois (environ 600 000), etc.

\section{La pêche piroguière, une activité commerciale mondialisée}

Jugée primitive, peu productive, peu évolutive durant la période coloniale $^{[10]}$, la pêche piroguière connaît pourtant de profondes évolutions depuis une cinquantaine d'années, comme le démontre l'aug-

[8] Le panel du GATT qui s'en suivit rejettera la demande américaine. [9] Selon l'Observatoire économique des pêches au Sénégal, le reste de la production provient de l'armement industriel national (48 000 tonnes) et de la flotte étrangère (12 300 tonnes).

[10] J.-P. Chauveau, E. Jul-Larsen et C. Chaboud, Les pêches piroguières en Afrique de l'Ouest : pouvoirs, mobilités, marchés, Karthala, Paris, 2000. mentation des débarquements (de 45000 tonnes en 1944 à environ 380000 tonnes en 2002).

Ce développement rapide est certes lié à la richesse halieutique des eaux sénégalaises, mais il s'explique surtout par le dynamisme des sociétés halieutiques et plus particulièrement des pêcheurs qui ont su adopter, en les adaptant à leur contexte, des innovations technologiques venues de l'extérieur. La quasi-totalité des 11000 pirogues recensées sur le littoral est équipée de moteur hors-bord ; certaines d'entreelles mesurent plus de 20 mètres de long et peuvent embarquer jusqu'à 15 tonnes de poissons. De même, les engins de captures et les techniques de pêche ont évolué : l'introduction dans les années 1970 de la senne tournante a permis un accroissement sans précédent des débarquements ${ }^{[11]}$. De même, l'utilisation généralisée de moyens de conser- 
vation à bord des pirogues dans les années 1980, puis d'apparaux de navigation et de repérage (sondeur et GPS) dans les années 1990 ont permis d'étendre l'espace de production des pêcheurs et de favoriser le développement de pratiques de pêche de plus en plus compétitives.

Ce développement a de plus été largement stimulé par le dynamisme de la filière de commercialisation. Depuis les années 1960, la demande nationale en poisson ne cesse de croître du fait de l'évolution des habitudes alimentaires, de la croissance démographique et de l'urbanisation. Ce dynamisme reste également lié au secteur de la transformation artisanale qui, en absorbant environ $40 \%$ des débarquements, permet d'approvisionner les populations les plus isolées tout en créant de nouveaux échanges commerciaux avec les pays limitrophes. Enfin, cette vitalité se retrouve aux niveaux des exportations vers les marchés européens et asiatiques. En effet, celles-ci ne cessent d'augmenter depuis les années 1970 du fait de l'internationalisation du marché des produits de la mer et de mesures fiscales, douanières et financières plus spécifiques ${ }^{[12]}$. Le Sénégal se classe ainsi au troisième rang des 20 plus grands exportateurs de poisson à destination de l'Europe ${ }^{[13]}$.

La pêche piroguière constitue désormais une activité commerciale dynamique et fortement mondialisée. Cette évolution significative a entraîné l'introduction massive de capitaux exogènes aux sociétés halieutiques : de plus en plus d'unités de pêche sont armées par des capitaux provenant de mareyeurs nationaux et internationaux (mauritaniens notamment) ou de fonctionnaires et de commerçants qui investissent dans la pêche pour faire fructifier leurs gains. En plus de favoriser l'accroissement de l'effort de pêche, cette situation engendre de profondes mutations dans l'organisation des unités de production. Fondés jusqu'alors sur la cellule familiale, l'armement de l'unité de pêche et l'organisation du travail tendent progressivement à être fondés sur des structures capitalistes. Certaines unités de pêche piroguière s'apparentent ainsi désormais davantage à une activité de type semi-industrielle ${ }^{[14]}$.

Dans ce contexte, l'application du concept de géosystème halieutique au cas de la pêche piroguière démontre que son développement est désormais confronté à de nouveaux enjeux et à de multiples conflits.

\section{Menaces environnementales et conflits sociaux}

L'espace de production apparaît fortement dégradé, dilaté et convoité. Les eaux sénégalaises sont en effet caractérisées par une importante diminution des ressources halieutiques : les biomasses des eaux côtières sont cinq fois moins importantes que dans les années 1950. Cet état de surexploitation, qui affecte tous les types
[11] M. Kébé et M. Deme, Filière pêche artisanale : rentabilité, exportations et consommation locale, ISRA, Dakar, 1996.

[12] Telles que la convention de Lomé qui autorise les produits halieutiques des pays de l'ACP à pénétrer le marché européen en franchise de douane ou la dévaluation du franc CFA en 1994, ce qui a accru la valeur marchande des produits sur les marchés extérieurs.

[13] UICN, Affaires de commerce : pêche au Sénégal et en Mauritanie, 2006,

http://www.iucn.nl/nederland s/publicaties/publicaties/Affa ires\%20de\%20commerce! \%20P\%EAche.pdf.

[14] S. Le Roux, Pêche et territoires au Sénégal, thèse de géographie, université de Nantes, 2005. 
de ressources côtières, a longtemps été mis en relation avec l'effort de pêche industrielle, et plus particulièrement celui des flottes étrangères. Or, il est désormais reconnu que la pêche piroguière participe aussi à cette diminution. Les innovations technologiques et l'intégration rapide à l'économie de marché ont en effet profondément modifié les rapports qu'entretiennent les pêcheurs avec les ressources. Le poisson est devenu un produit commercial et les pêcheurs, contraints par de nouvelles obligations de rentabilité économique, ont accru leurs pressions sur celui-ci en développant des pratiques de pêche de plus en plus compétitives. Cette accentuation de la pression est également à mettre en corrélation avec l'extraversion croissante de la filière de commercialisation, puisque les espèces qui montrent des signes visibles de surexploitation sont celles qui sont principalement destinées à l'exportation. Cette situation est à l'origine d'une exacerbation des tensions sociales selon trois principaux types : entre pêcheurs artisans et autorités gouvernementales transfrontalières ; entre pêcheurs artisans et pêcheurs industriels ; entre pêcheurs artisans de différentes origines.

Cette diminution des ressources côtières, associée au développement de la commercialisation des espèces démersales ${ }^{[15]} \mathrm{du}$ fait de l'accroissement de la demande des marchés extérieurs, a conduit de nombreux pêcheurs à déployer leur effort vers de nouveaux stocks situés plus au large ou dans les eaux des pays limitrophes. Par exemple, nombre d'unités de pêche démersale, communément appelées, « pirogues glacières », dessinent un espace de production qui va de la Mauritanie à la Sierra Léone, tandis que certaines unités de sennes tournantes de Saint-Louis vont régulièrement exploiter les ressources pélagiques des eaux mauritaniennes. Malgré l'octroi de licences de pêche par les gouvernements voisins, cette situation génère des tensions politiques entre les pays de la sous-région, et plus particulièrement avec la Mauritanie. Plusieurs unités de pêche n'ayant pas de licences ont été arraisonnées par les gardes côtes mauritaniens, mais malgré la confiscation du matériel et de fortes amendes, les pêcheurs

[15] Espèces vivant à proximité du fond des mers sans être totalement dépendantes de celui-ci. Certaines d'entre elles, comme les dorades, les capitaines, les rougets ou les mérous, sont en voie de disparition.

[16] une centaine par an selon la presse sénégalaise. incriminés déclarent être prêts à repartir illégalement exploiter ces eaux. Ils justifient leurs actes par le fait qu'elles sont plus abondantes que les eaux sénégalaises, qu'elles ne sont pas exploitées par les pêcheurs nationaux et que les espèces migrent le long des côtes ouestafricaines. D'autre part, la dilatation de l'espace de production des unités de pêche piroguière a accru les situations conflictuelles avec la pêche industrielle. Ces dernières années ont été marquées par une augmentation des collisions entre pirogues et chalutiers causant la mort de plusieurs pêcheurs artisans ${ }^{[16]}$. Par ailleurs, la raré- 
faction des ressources est à l'origine d'une exacerbation des tensions entre pêcheurs artisans. Elles sont tout d'abord liées à l'utilisation, dans un même espace, de techniques de pêche peu compatibles entre elles, ainsi qu' au développement de pratiques peu respectueuses de certaines règles élémentaires telles que la propriété d'usage, la capture des alevins et des juvéniles ou l'utilisation de pratiques néfastes (pêche à l'explosif).

L'analyse des pôles structurants du géosystème halieutique sénégalais démontre que des tensions existent également à terre. Tout d'abord, au niveau des plages de débarquements, on remarque des heurts de plus en plus fréquents entre les pêcheurs traditionnellement ancrés dans le monde de la pêche et ceux qui viennent d'y arriver. En effet, l'armement d'unités de pêche par des capitaux exogènes aux communautés de pêcheurs a entraîné l'apparition d'une catégorie de pêcheurs salariés. Or, ceux-ci sont souvent accusés d'être à l'origine de pratiques peu respectueuses de la pérennité des ressources, mais aussi de conduites néfastes au niveau des plages de débarquements (collision entre pirogues, vols). Toutefois, si ces accusations semblent dans certains cas avérées, elles sont aussi liées à des jeux identitaires.

On note également des tensions de plus en plus vives entre les pêcheurs migrants et autochtones. Les modes d'occupation de l'espace littoral par les pêcheurs artisans se caractérisent par d'importants phénomènes migratoires : nombre de pêcheurs quittent leur village natal durant une grande partie de l'année pour s'installer dans d'autres centres de pêche afin de réunir les conditions écologiques et économiques les plus favorables à la pratique de leur métier. Or, ces stratégies spatiales soulèvent de plus en plus de tensions pouvant atteindre de forts degrés de violence, comme l'illustre l'exemple suivant.

\section{Conflits entre pêcheurs autochtones et migrants}

Situé au nord de la capitale sénégalaise, le centre de pêche de Kayar, le troisième du pays, est une des plus anciennes destinations pour la communauté des pêcheurs guet-ndariens (un quartier de Saint-Louis), où se regroupent plus de deux milles pirogues. Cependant, la présence de ces pêcheurs migrants est mal acceptée par les pêcheurs autochtones, voire plus généralement par la population locale, ce qui crée d'importantes tensions. Par exemple, en juin 2005, des heurts opposent sur la plage ces deux communautés. Les Kayarois s'en prennent aux pirogues saint-louisiennes et en brûlent quelques-unes malgré la résistance des Guet-ndariens. Devant cette violence, les forces de l'ordre interviennent, mais les Kayarois décidés à chasser les Saint-Louisiens de leur village s'en prennent aux 
gendarmes, qui ripostent alors avec leurs armes. Les heurts vont alors durer près d'une heure faisant une vingtaine de blessés et un mort, un jeune pêcheur d'une vingtaine d'années. Illustrant de la façon la plus violente les tensions entre pêcheurs artisans, ces heurts communautaires ne sont pas nouveaux et reviennent en effet sporadiquement $(1953,1985,2001)$.

Une étude sur ces conflits évoque des raisons liées à l'utilisation de certains engins de captures, et plus particulièrement la pratique du filet dormant par des Saint-Louisiens. Cependant, elle souligne que ceci ne constitue "qu'un facteur de déclenchement et de pérennisation des hostilités ». Les origines de ces heurts résultent davantage « de différences comportementales et de raisons sociologiques [...], d'une conception diamétralement opposée de l'accès à la mer et à ses ressources » développée traditionnellement par chacune des communautés. "Les Kayarois, à l'origine paysans-pêcheurs, projettent sur la mer en face de leur village leur conception terrienne de l'appropriation du terroir et considèrent cette zone de pêche comme leur bien exclusif. [...] Les Guet-ndariens, quant à eux, pratiquent exclusivement la pêche et sont fiers de leur ancienne tradition de pêcheurs migrants. Pour eux, le libre accès aux zones de pêche est un droit inaliénable et une condition vitale à la survie de leur tradition, de leur mode d'organisation socio-économique ${ }^{[17]} »$. De plus, la sédentarisation d'un nombre croissant de pêcheurs migrants et d'autres éléments sous-jacents (querelles de voisinage, luttes pour le pouvoir, actions de certains agitateurs) suscitent parmi les Kayarois des réflexes collectifs pour défendre leur territoire. Enfin, ces conflits sont à mettre en relation avec la raréfaction de certaines espèces (notamment celles dites nobles) qui a ravivé la dualité entre ces deux communautés.

On s'aperçoit d'ailleurs que ces tensions, jusqu'à présent limité au cas de Kayar, tendent à se diffuser dans d'autres centres de pêche du pays. Dans un contexte de diminution des ressources, les pêcheurs autochtones affichent de plus en plus ouvertement leur volonté de restreindre la présence des pêcheurs migrants dans les eaux qui bordent leur village.

L'exemple de la pêche piroguière sénégalaise démontre qu'il existe

[17] CRODT, Conflit de Kayar, analyse du Centre de recherche océanographique de Dakar-Thiaroye, document interne, 1985, p. 4. une multitude de conflits autour de l'activité, tant en termes d'exploitation que de distribution ou de consommation et que ceux-ci peuvent parfois prendre des formes violentes. Ceci nous conduit à aborder les diverses solutions multiscalaires mises en œuvre pour à la fois réguler l'accès aux ressources et tenter d'atténuer ces rivalités. 


\section{Des tentatives diverses de régulation de l'activité pêche à plusieurs échelles}

Pour tenter de gérer au mieux l'activité halieutique, diverses politiques sont mises en place à différents niveaux : international, avec des organisations intergouvernementales comme l'Organisation des Nations unies pour l'alimentation et l'agriculture (FAO) ; national avec l'État sénégalais par exemple... Néanmoins, pour un certain nombre de pêcheurs, ces mesures de régulation restent insatisfaisantes car elles ne couvrent pas l'activité dans sa globalité, notamment sa dimension socioculturelle.

\section{Une régulation internationale de l'activité pêche centrée sur l'écosystème}

Selon les dernières estimations de la FAO, plus de $75 \%$ des pêcheries mondiales sont actuellement en état de surexploitation ${ }^{[18]}$. L'état précaire de la ressource, conjugué à l'intensification de l'exploitation et de la commercialisation nécessitent de prendre des initiatives pour préserver l'écosystème marin et réguler l'intensité de cette activité. Les spécificités des ressources expliquent les difficultés de gestion, car les hommes n'ont aucun contrôle sur leur distribution, leur migration ou leur productivité naturelle. Schématiquement, il existe deux grands types d'instruments de régulation et de contrôle de l'activité, l'un portant plus sur les ressources (l'écosystème), l'autre sur les moyens de production, (le sociosystème). Cette approche géosystémique de la régulation combine des facteurs internes (stocks, effort de pêche, marché, réglementation, etc.) et externes (environnement aquatique, activités concurrentes, contexte politico-économique, politiques d'aménagement, etc. $)^{[19]}$.

En matière de gestion internationale des conflits, de nombreux efforts sont consentis sur l'environnement marin et sur les écosystèmes côtiers. Une première mesure est instaurée dès 1995 par la FAO avec l'élaboration d'un «Code de conduite pour une pêche responsable » qui « définit les premiers principes et normes internationales de comportement pour garantir des pratiques responsables en vue d'assurer la conservation, la gestion et le développement des ressources bioaquatiques, dans le respect des écosystèmes et de la biodiversité $^{[20]} \gg$. Ces dernières années, diverses démarches de régulation sont en effet mises en œuvre pour protéger des échantillons représentatifs en termes de milieu (aires marines protégées, écotourisme) et/ou à valo-
[18] http://www.fao.org/fi. [19] G. Biais, J. Catanzano, B. Mesnil et H. Rey, Système halieutique. Un regard différent sur les pêches, IFREMER, Paris, 1997. [20] http://www.fao.org/fi/ agreem/codecond/ codeconf.asp. 
riser de manière sélective certaines espèces en orientant le comportement d'achat des consommateurs (écolabellisation, éco-étiquetage).

À titre d'exemple, en 1998, la société américaine Unilever (premier acheteur mondial de poissons) signe un accord avec le Fonds mondial pour la nature (WWF) afin de mettre en place un Conseil de surveillance marine (MSC), suivi d'un écolabel intitulé « Fish Forever». Or, aujourd'hui, cette initiative privée de certification ne touche qu'une petite fraction d'espèces (homard d'Australie, saumon d' Alaska, hoki de Nouvelle-Zélande, etc.), produites et consommées à une très large majorité par les pays du Nord. En outre, cet écolabel implique de forts risques de discrimination pour les pays du $\mathrm{Sud}^{[21]}$ :

- un cantonnement dans un rôle de producteurs sous dépendance de firmes multinationales (notamment Wal Mart, leader de la grande distribution américaine) ;

- des stratégies de segmentation des marchés par de nouvelles techniques de différenciation marketing ;

- une tendance à une relative standardisation des espèces par rapport aux pêcheries artisanales et diversifiées.

Cet exemple de certification privée traduit la relative ambiguïté de ces démarches qui demeurent surtout centrées sur la régulation des conflits autour de l'accès aux ressources. À une échelle plus fine, l'exemple du Sénégal démontre à nouveau toute la complexité liée à la gestion de cette activité.

\section{La gestion problématique \\ de la ressource à l'échelle sénégalaise}

Conscient du rôle de la pêche piroguière dans la raréfaction des ressources halieutiques, l'État sénégalais mène actuellement des réflexions afin de réguler l'effort de pêche. Symbole d'un nouveau modèle de gestion concertée des ressources halieutiques en Afrique de l'Ouest, cette politique prévoit de mettre fin au principe du libre accès aux ressources, considéré comme une des principales raisons du fort accroissement de l'effort de pêche. Cependant, la mise en place de nouveaux modes d'accès aux ressources s'avère problématique. Elle va tout d'abord à l'encontre de la perception qu'ont les pêcheurs artisans de la mer, ceux-

[21] Forum mondial des pêcheurs et des travailleurs de la pêche (FMPTP), La pêche en eau trouble? Propositions pour une gestion durable des ressources marines, Éditions Charles Léopold Mayer, Paris, 2004. ci la considérant comme espace libre de toutes contraintes autres que celles imposées par la nature. Ensuite, elle risque d'aggraver les conflits actuels, voire d'engendrer de nouvelles tensions sociales.

Les processus de concertation destinés à établir cette nouvelle législation donnent lieu à de nouveaux jeux identitaires au sein des sociétés halieutiques. Dans les réunions de concertation, la distinction entre pêcheurs artisans professionnels et non-professionnels, sous-entendant 
la division entre pêcheurs « traditionnels » et pêcheurs « salariés », est de plus en plus fréquente. On remarque ainsi une plus grande volonté des pêcheurs dits traditionnels de revendiquer une appartenance ethnique Lébou $^{[22]}$, ce qui leur permet de pouvoir naturellement prétendre à des droits d'accès à la ressource. Des jeux de pouvoirs apparaissent aussi au sein des organisations socioprofessionnelles qui interviennent dans cette concertation. Qu'elles soient nationales ou locales, celles-ci sont le plus souvent entre les mains d'hommes de pouvoir qui défendent leurs propres intérêts, ce qui tend à accroître les tensions entre pêcheurs.

Ces nouveaux modes de régulation sont à l'origine d'un mécontentement de plus en plus grand à l'égard du gouvernement et de l'émergence de revendications nationalistes. Un nombre important de pêcheurs artisans n'admettent pas que l'État veuille contrôler, voire réduire, leur effort de pêche au nom de la préservation des ressources alors que, dans le même temps, celui-ci continue d'attribuer des licences de pêche à des navires industriels étrangers. Ce mécontentement est relayé par certaines organisations socioprofessionnelles telles que le Collectif national des pêcheurs artisans sénégalais (CNPS) qui, dès sa création en 1987, s'est clairement déclaré contre ces accords de pêche. De plus en plus d'acteurs de la société civile remettent également en cause la signature de tels accords. Selon Dahou et Deme, ils n'ont plus lieu d'exister pour les espèces démersales côtières puisque la flotte nationale est désormais en mesure de les exploiter pleinement ${ }^{[23]}$. Lorsque ces accords ont été mis en place il y a une trentaine d'années, leur justification reposait principalement sur le fait que le Sénégal disposait de ressources abondantes que la flotte nationale n'était pas en mesure d'exploiter de façon optimale. Or ce n'est plus cas aujourd'hui. Toutefois, étant donné l'importance des contreparties financières liées à ces accords de pêche ${ }^{[24]}$, le gouvernement peut difficilement les abandonner. Il devra cependant faire un choix, surtout que les négociations pour la reconduite des prochains accords, prévus en 2007, coïncident avec les échéances présidentielles. La question du contrôle et de la gestion des ressources halieutiques risque donc d'être au cœur des prochains débats politiques. Cette situation est d'autant plus préoccupante que le ressentiment envers l'Occident apparaît de plus en plus pesant au sein de la société sénégalaise.

L'État entend rompre avec le fait que le métier de pêcheur soit considéré comme un héritage familial. Mais cette mesure risque de profondément déstabiliser les sociétés halieutiques. En effet, malgré les évolutions récentes, la pêche demeure une activité familiale. En outre, ces sociétés sont caractérisées par un taux d'accroissement naturel supérieur à la moyenne nationale. Jusqu'à présent, la pêche, par son
[22] Les Lébou, à l'instar des Guet-ndarien de SaintLouis, représentent une des grandes communautés de pêcheurs de l'Afrique de l'Ouest.

[23] K. Dahou et M. Deme, Accords de pêche UE-Sénégal et commerce international : respects des réglementations internationales, gestion durable des ressources et sécurité alimentaire, ENDA DIAPOL, Dakar, 2002.

[24] Environ 42 milliards de francs CFA pour une durée de quatre ans. 
dynamisme économique, protège les jeunes générations des familles de pêcheurs du phénomène de paupérisation dont est victime une grande partie de la population sénégalaise. Mais si ces jeunes ne peuvent plus pratiquer le métier de leurs parents, ils risquent de venir « gonfler» le nombre de chômeurs qui tentent de survivre dans l'agglomération dakaroise, ce qui risque d'aggraver les problèmes sociaux. Ces nouveaux moyens de régulation doivent également prendre en compte que l'élaboration d'un contexte favorable à la pratique d'une pêche destinée à l'exportation a entrainé un important report de l'effort de pêche vers les espèces démersales, ce qui a provoqué une diminution quantitative et qualitative des produits halieutiques sur le marché local ainsi qu'une augmentation des prix ; or, «dans un pays où le poisson pourvoit à $75 \%$ des besoins en protéines animales, cette évolution fait peser un grave danger sur la sécurité alimentaire ${ }^{[25]} »$.

Face à ces nouveaux enjeux, il convient donc d'être extrêmement prudent dans la mise en place de cette législation car elle risque d'accroître les tensions au sein des sociétés halieutiques, voire plus généralement de nuire à la stabilité sociale et politique du Sénégal. Cet exemple traduit la relative ambiguïté des mesures de régulation des conflits qui ne prennent pas suffisamment en considération les coûts sociaux et humains de l'activité. Dans ce contexte, de nombreux acteurs de la filière ont décidé il y a une vingtaine d'années de se regrouper de manière transnationale afin de réfléchir à d'autres modes de gestion.

\section{Une tentative de gestion " alternative ": l'altermondialisation halieutique}

Dès les années 1980, confrontés aux stratégies spéculatives du secteur de la pêche ainsi qu'à une grave crise de surexploitation de la ressource, de nombreux pêcheurs artisans traditionnels - essentiellement des pays du Sud - décident de réagir. Parallèlement à la conférence mondiale sur la pêche organisée par la FAO à Rome en 1984, ces pêcheurs se sentant marginalisés, aussi bien par les grandes organisations intergouvernementales émanant des Nations unies que par leurs États respectifs, décident de se réunir pour tenter de consolider leurs organisations et de structurer leurs réseaux. Un collectif international d'appui aux pêcheurs artisans est donc créé dès 1986 afin de donner

[25] K. Dahou et M. Deme, op. cit.

[26] A. Le Sann, « De Rome à Loctudy : la naissance difficile d'un mouvement international des pêcheurs artisans ", Cahiers Nantais, $n^{\circ} 55-56,2001$, p. 281-288 plus de lisibilité à ce mouvement alternatif de l'halieutique. À la suite de plusieurs rencontres sur une dizaine d'années (Rome en 1984, Québec en 1995), ces mêmes producteurs artisans finissent par constituer, en 1997, à Delhi le Word Forum of Fishworkers (WFF) dont l'objectif principal est la défense «de leurs droits fondamentaux, de la justice sociale et de leur culture ${ }^{[26]} »$. 
Ces altermondialistes de l'halieutique ${ }^{[27]}$, dont faisaient partie activement les membres du CNPS, critiquent unanimement la vision politique libérale du secteur, qui conduit au développement d'activités productivistes et industrielles déterritorialisées. Le poisson y est avant tout considéré comme "une denrée marchande qui doit être source de profits et non pas une ressource qu'il importe de préserver pour garantir des moyens d'existence aux populations ${ }^{[28]} \gg$. Selon eux, cette vision entraîne par conséquent un délaissement, voire un abandon, de la pêche artisanale et traditionnelle, alors que celle-ci contribue pourtant à une relative stabilité socio-spatiale ${ }^{[29]}$ de ces populations.

Les membres de ce WFF participent pleinement à la régulation des conflits et tentent de promouvoir des activités halieutiques diversifiées, plus responsables et plus durables. Ces altermondialistes proposent donc toute une série de mesures et de nouvelles pistes de réflexion afin de favoriser la naissance d'un nouvel ordre halieutique international. À titre de propositions alternatives, il nous semble que la mise en place d'un commerce équitable des produits de la mer s'appuyant sur une démarche géosystémique, (c'est-à-dire depuis la capture jusqu'à la consommation et en synergie avec les démarches de régulations écosystémiques décrites précédemment), mérite d'être approfondie. Ce type de commerce semble en effet insister davantage sur la dimension socio-spatiale de l'activité halieutique et pourrait permettre de résoudre certaines insuffisances en termes de gestion des conflits. Il privilégie en outre une répartition et une valorisation de la ressource plus égalitaires tout en assurant de meilleures conditions de vie et de travail pour les divers opérateurs de la filière, tant en termes de rémunération (prix minimum) que d'identité (savoir-faire).

\section{Conclusion}

Au travers d'une démarche méthodologique fondée sur le géosystème halieutique, nous avons montré que la filière pêche s'est globalement mondialisée tout en provoquant de profondes recompositions contradictoires : atomisation des espaces de production versus concentration des espaces de consommation et de commercialisation. Ces modifications spatiales se répercutent également à d'autres niveaux d'échelles comme le montre le cas de la pêche piroguière sénégalaise. Elles contribuent également à produire de nombreux antagonismes autour de la ressource : menaces environnementales, conflits sociaux, concurrences commerciales, insécurité alimentaire, etc. Pour contrecarrer ces rivalités, différentes mesures de gestion sont mises en place à diverses échelles (surtout internationales et étatiques). Mais ces poli-
[27] J. Noël, Les enjeux géographiques de l'altermondialisation dans la production et les échanges de produits halieutiques (et aquacoles), IGARUN, Nantes, mémoire de DEA de géographie, 2004.

[28] FMPTP, op. cit. [29] J.-P. Corlay, 2004 op. cit. 
tiques de régulation de la ressource privilégient essentiellement des préoccupations environnementales, alors que des solutions « alternatives » sont envisagées par certains acteurs afin de mieux intégrer les dimensions socioculturelles de l'activité.

Dans un contexte globalisé, il semble qu'il n'y ait pas de régulation idéale des conflits autour des ressources halieutiques. Néanmoins, face aux stratégies de gestion « curative » utilisées en temps de crise ou de gestion «préventive » trop ambitieuses, ou face aux stratégies libérales ou conservationnistes, Cury et Roy préconisent une gestion davantage "réactive » qui s'adapte à ces mêmes ressources ${ }^{[30]}$. Bien souvent, différents systèmes de gestion sont mis en place et se complètent. Mais ces derniers doivent être pensés différemment selon les contextes spatiaux (c'est-à-dire biologique, économique, social, culturel et politique) dans lesquels ils s'insèrent. Selon nous, l'activité de pêche se doit d'être administrée dans sa globalité ; elle nécessite par conséquent la mise en place d'une véritable « gestion alternative intégrée $^{[31]} »$, garante d'une filière halieutique à la fois écologiquement satisfaisante, économiquement rentable et surtout socialement équitable, et ce à toutes les échelles et sur le long terme. 